\title{
Circular RNAs in acute myeloid leukemia
}

\author{
Vijendra Singh ${ }^{1}$, Mohammed Hafiz Uddin², Jeffrey A. Zonder ${ }^{1}$, Asfar S. Azmi ${ }^{2}$ and \\ Suresh Kumar Balasubramanian ${ }^{1 *}$ (1)
}

\begin{abstract}
Although mechanistic studies clarifying the molecular underpinnings of AML have facilitated the development of several novel targeted therapeutics, most AML patients still relapse. Thus, overcoming the inherent and acquired resistance to current therapies remains an unsolved clinical problem. While current diagnostic modalities are primarily defined by gross morphology, cytogenetics, and to an extent, by deep targeted gene sequencing, there is an ongoing demand to identify newer diagnostic, therapeutic and prognostic biomarkers for AML. Recent interest in exploring the role of circular RNA (circRNA) in elucidating AML biology and therapy resistance has been promising. This review discerns the circular RNAs' evolving role on the same scientific premise and attempts to identify its potential in managing AML.
\end{abstract}

Keywords: Acute myeloid leukemia, Non-coding RNA, Circular RNA

\section{Introduction}

Acute myeloid leukemia (AML) is characterized by clonal expansion of myeloid blasts in the bone marrow and peripheral blood. AML was originally classified based on the morphological appearance of the myeloid blasts [1], and subsequently according to whether recurrent characteristic chromosomal abnormalities (with specific resultant gene fusions and point mutations in different myeloid genes) were present $[2,3]$. As the mechanistic underpinnings associated with these genetic alterations have been elucidated, the clinical utility of detailed molecular characterization of AML has extended beyond simply providing a framework for disease classification, now permitting prognostication, response monitoring, and - increasingly - patient-specific treatment decisions. Despite these advancements, the overall prognosis remains dismal [5-year overall survival 28.7\%] [4]. Inherent and acquired resistance to therapy contributes to high rates of early disease relapse and death, even in patients not initially identifies as having "high-risk" molecular

*Correspondence: balasubs@karmanos.org

1 Department of Oncology, Karmanos Cancer Institute/Wayne State University, 4100 John R, HWCRC 740.2, Detroit, MI 48201, USA

Full list of author information is available at the end of the article features, underscoring the need for innovative, predictable, and robust alternative biomarkers.

Recent advances in high-throughput sequencing demonstrated that the majority of mRNA $(\sim 75 \%)$ of the entire human transcriptome do not get translated into proteins and are called non-coding RNA (ncRNA) [5]. ncRNAs are divided based on transcript sizes into small ( $<200$ nucleotides) and long ( $>200$ nucleotides) RNAs (sncRNAs and lncRNAs, respectively). While themselves not being translated into proteins, ncRNAs regulate gene expression by regulating transcription, post-transcriptional modifications, and translation [6,7]. Altered ncRNAs may have a pathogenic role in the development of different cancers, including AML, in which they have been implicated in both leukemogenesis and drug resistance [8].

CircRNA is a single-stranded ncRNA which, due to a closed-loop structure secondary to a phosphodiester bond between the $3^{\prime}$ and $5^{\prime}$ ends, as well as the absence of a $3^{\prime}$ polyadenyl tail, has increased stability and a longer half-life compared to linear RNA [9]. The same structural uniqueness also makes them resistant to nucleases [10]. CircRNAs are produced from canonical or non-canonical splicing of any of several parts of the primary transcripts, including exon, intron, $3^{\prime}$ and $5^{\prime}$ untranslated regions,

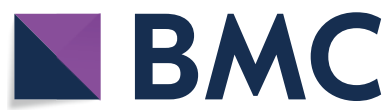

(c) The Author(s) 2021. Open Access This article is licensed under a Creative Commons Attribution 4.0 International License, which permits use, sharing, adaptation, distribution and reproduction in any medium or format, as long as you give appropriate credit to the original author(s) and the source, provide a link to the Creative Commons licence, and indicate if changes were made. The images or other third party material in this article are included in the article's Creative Commons licence, unless indicated otherwise in a credit line to the material. If material is not included in the article's Creative Commons licence and your intended use is not permitted by statutory regulation or exceeds the permitted use, you will need to obtain permission directly from the copyright holder. To view a copy of this licence, visit http://creativecommons.org/licenses/by/4.0/. The Creative Commons Public Domain Dedication waiver (http://creativeco mmons.org/publicdomain/zero/1.0/) applies to the data made available in this article, unless otherwise stated in a credit line to the data. 
intergenic regions, and sometimes from antisense RNAs $[11,12]$. Based on the primary transcript region involved in splicing, the resultant circRNA can be exonic circRNA, intronic circRNA, intron-exon circRNA, and intergenic circRNA [9, 11-13]. Most described circRNAs are of exonic subtype. Fusion circRNA has been identified in malignancies associated with chromosomal translocations [14]. Various mechanisms of circRNA biogenesis are demonstrated in Fig. 1. The majority of circRNAs are located in the cytoplasm, and the number of circRNA transcripts is enriched relative to the corresponding amount of linear RNA derived from a particular gene [15]. CircRNA expression is tissue and developmental stage-specific $[13,16]$. CircRNA regulates gene expression by directly or indirectly altering transcription and translation through several mechanisms [17], including by sponging or decoying microRNA (miRNA), thereby reducing miRNA-mediated degradation of messenger

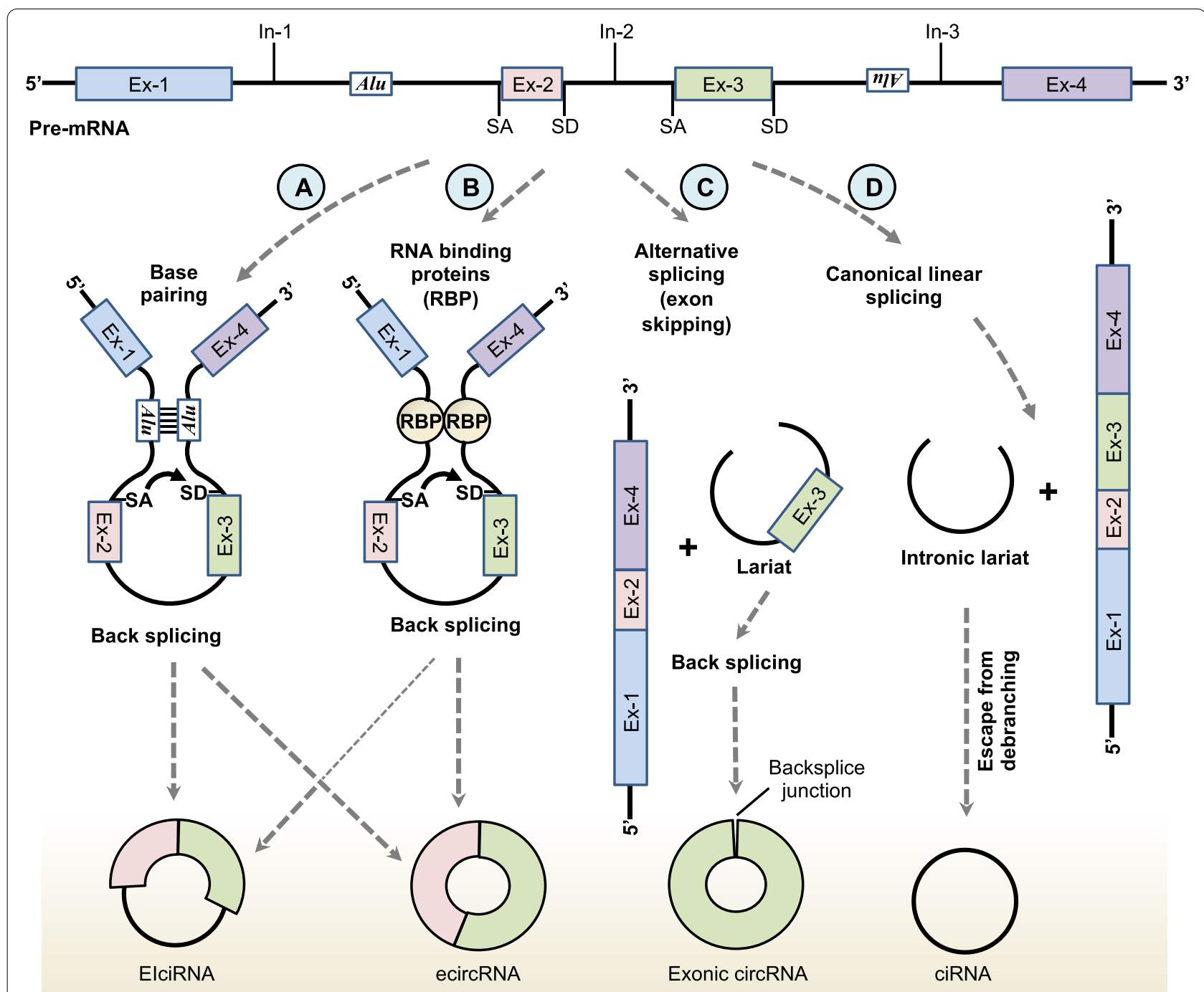

Fig. 1 The biogenesis of diverse circRNA. There are several mechanisms of circRNA biogenesis. A Intron pairing mediated circularization. B RBP mediated circularization. Backsplicing of RNA is favored by long flanking introns, the presence of inverted repeat elements (e.g., Alu elements), and trans-acting RNA binding proteins (A, B) [70]. The base pairing between different introns brings together upstream splice acceptor (SA) and downstream splice donor (SD) sites, leading to backsplicing. During backsplicing, SD attacks SA, which results in exon-intron circRNAs (ElcircRNAs) or exonic circRNAs. C Alternative splicing mediated circularization (lariat-driven). The circRNAs also can originate from alternative splicing intermediates called lariat precursors. In this process, RNA is spliced linearly but skips specific exon. D Canonical linear splicing mediated circularization (intronic lariat-driven). Here intronic lariat precursors that avoid the debranching step make circRNA exclusively contain introns. Ex, exon; In, intron; RBP, RNA binding protein; SA, splice- acceptor site; SD, splice- donor site; ElcircRNAs, exon-intron circRNAs; Alu, Alu inverted repeat elements; BSJ, backsplice junction; ciRNA, circular intronic RNA 
RNA (mRNA) [18]. CircRNA can also directly interact with proteins and modulate their function [19]. For example, circRNA binds and decoys RNA binding proteins (RBPs) [20], and it also facilitates co-localization of enzymes by serving as a protein scaffold [21]. Though circRNAs are considered to be one type of ncRNA, recent studies have confirmed that they can be translated in some cases [22, 23].

Long non-coding RNA (lncRNA) is another ncRNA produced during transcription by RNA polymerase II and is expressed in a tissue-specific manner [24]. In contrast to circRNA, most lncRNAs are localized to the nuclear compartment. Otherwise, circRNA and lncRNA share certain common properties (i.e., being non-coding, highly conserved, and having a relatively long halflife) and functions (e.g., sponging miRNAs). Both RNA species have specific mechanisms of action relevant to pathogenesis of disease, including leukemia [25, 26].

CircRNA, specifically, has been implicated in Alzheimer's disease, diabetes mellitus, cardiovascular diseases, and many other conditions, including but not limited to cancer [27]. Aberrant expression of circRNAs has been implicated in various steps of tumorigenesis, metastasis, and drug resistance [28]. The significance of circRNA in cellular homeostasis and tumorigenesis, especially considering its abundance in body fluids, including blood, makes it ideal as a potential prognostic and diagnostic biomarker [29-31]. This review summarizes several circRNAs with altered expression reported in AML, their role in disease biology, and their diagnostic, prognostic, and therapeutic potential.

CircRNA as a biomarker for diagnosis and prognosis in AML Multiple studies have explored the potential use of circRNAs in the diagnosis and prognostication of AML. Variable expression of specific circRNAs studied in different model systems have been found to be prognostic in AML. Yi et al. demonstrated upregulation of circ-VIM by means of reverse transcriptase and quantitative polymerase chain reaction (RT-qPCR) in AML patients' bone marrow samples compared to healthy controls [32]. Based on circ-VIM levels, AML patients can be divided into two cohorts: low and high expressers. Circ-VIM expression correlated with certain clinical features, such as white blood cell (WBC) counts and French-American-British (FAB) AML morphologic subtypes. In multivariate analysis, high circ-VIM expressers showed shorter leukemiafree and overall survival. The accuracy of circ-VIM as a biomarker was demonstrated by the area under the receiver-operating characteristic curve (AUC of ROC) of 0.741 comparing AML patients to healthy controls. In this study, the expression of parental gene VIM positively correlated with circ-VIM expression. Though VIM expression's prognostic significance was not assessed in this study, authors alluded to the possibility of circ-VIM as a tumor promoter.

Similarly, in another study, circ-Foxo3 was downregulated in de novo AML patients, and circ-Foxo3 expression positively correlated with Foxo3 gene expression [33]. ROC analysis suggests circ-Foxo3 expression may have diagnostic utility in AML. Conclusions regarding its use as prognostic factor were less clear, as high Foxo3 gene expression was associated with a better prognosis but the circ-Foxo3 expression was not.

In another study, microarray-based analysis revealed 147 up and 317 downregulated circRNAs respectively in six newly diagnosed cytogenetically normal AML (CN-AML) patients with varying prognostic risk based on FLT3 and NPM1 mutation status compared to four healthy controls [34]. Further, a molecular signature based on the expression pattern of 5 specific circRNAs (2 upregulated; hsa_circi_0035381 and hsa circ_0049657, and 3 downregulated; hsa_circ_0001187, hsa_circ_0008078, and hsa_circ_0001947) which could distinguish between high and low-risk AML patients was identified. Hsa_circ_0004277, another circRNA downregulated in this cohort seems to be a promising diagnostic biomarker, with an AUC of ROC of 0.957 (AML patients versus healthy controls). More interesting perhaps was the finding that in a validation group of 107 AML patients at various stages of treatment, the significantly downregulated Hsa_circ_000427 expression observed at diagnosis was restored to control levels at remission, and then became downregulated again at disease relapse. Thus, serial measurement of hsa_circ_0004277 may potentially help in disease surveillance, as well as initial diagnosis.

Circ_009910 (exonic circRNA encoded by Mitofusin 2 gene on chromosome 1) and circ-ANAPC7 were upregulated in AML patients compared to controls with iron deficiency $[35,36]$. Circ_0009910 regulates cell cycle progression, proliferation, and apoptosis of leukemic cells by sponging miR-20a-5p. Poor-risk AML patients, particularly, had high circ_009910 levels, but the question of whether this had an impact on survival independent of the established factors used to define the poor-risk category was not evaluated.

More recently, whole-genome microarray comparing bone marrow mononuclear samples from extramedullary infiltrating (EMI) AML and non-EMI AML patients revealed 253 and 259 circRNAs to be up and downregulated, respectively, in EMI compared to non-EMI AML patients [37]. The authors identified 7 target genes regulated by 17 circRNAs (LRRK1, PLXNB2, OLFML2A, LYPD5, APOL3, ZNF511, and ASB2) associated with poor prognosis, and 2 genes (PAPLN and NRXN3) whose overexpression correlated with better prognosis. 
Another study demonstrated circ-PTK2 (hsa circ_104700/hsa_circ_0005273) to be upregulated in AML bone marrow samples compared to healthy controls [38]. Knockdown (KD) of circ-PTK2 suppressed proliferation and triggered AML cells' apoptosis by decreasing the expression of cyclin D1 and anti-apoptotic protein BCL-2 and increasing expression of pro-apoptotic protein Bax levels. In-vivo studies demonstrated that circ-PTK2 bound and sequestered miR-330-5p, leading to increased expression of FOXM1. High expression of circ-PTK2 was associated with shorter survival in AML patients, presumably due to its effect on leukemic cell proliferation and apoptosis.

In another study, microarray analysis performed on bone marrow samples from patients with acute lymphoblastic leukemia (ALL) and AML revealed differential expression of 10 specific circRNAs [39]. For example, hsa_circ_0012152 was found to be upregulated in AML compared to ALL (AUC of ROC 0.8625) and healthy controls (AUC of ROC 0.9773), while hsa_circ_0001857 was upregulated in ALL vs. AML (AUC of ROC 0.90911).

In summary, circRNAs have potential utility in AML as a diagnostic and prognostic biomarker and as a means of monitoring disease status and therapeutic response. The role of circRNA measurement to monitor disease status in the context of commercially available clinical measurable disease (MRD) monitoring remains to be determined. As MRD influences AML treatment decisions, it creates more opportunities to study circRNA further in this setting.

\section{Role of circRNA in elucidating AML biology}

Apart from being a diagnostic/prognostic biomarker in AML, the role of circRNA in AML biology and pathogenesis has also been explored (Table 1 and Fig. 2). There is a growing body of evidence that circRNA regulates gene expression and modulates various steps of leukemogenesis, including differentiation, proliferation, cell cycle transition, adhesion, and apoptosis.

\section{Regulation of apoptosis, cell cycle progression, and proliferation}

Although there are numerous factors contributing to leukemogenesis in AML, evading apoptosis seems to have a critical role [40]. A recent study demonstrated higher expression of circ-DLEU2 (Hsa_circ_0000488) in AML patients than healthy controls [41]. Higher circ-DLEU2 expression correlated with impaired apoptosis, increased cell proliferation in vitro, and accelerated tumor growth in vivo. Circ-DLEU2 upregulated PRKACB gene expression via sponging of miRNA-496. Note that PRKACB protein is the catalytic subunit of cyclic AMP-dependent protein kinase and is thus involved in regulating various cell signaling processes. While studies have reported the role of parent gene DLEU2 in leukemogenesis [42, 43], $D L E U 2$ expression and its effects were not investigated in this study.

In a study by Zhang et al., both circ_0000370 and its parent gene FLI-1 were found to be upregulated in AML patients [44]. Circ_0000370 expression was higher in FLT3-ITD patients than in FLT3 wild-type (WT) patients and was associated with a poor prognosis. In Vitro, FLT3ITD expression positively correlated with circ_0000370 expression, and quizartinib (a FLT3 inhibitor) downregulated circ_0000370 expression. Circ_0000370 regulated cell survival and apoptosis via increased $S 100 A 7 A$ expression by sponging miR-1299. In a different study, Sun et al. demonstrated that circMYBL2 (has_circ_0006332, parent gene $M Y B L 2$, a cell cycle checkpoint gene) expression to be approximately five-fold higher in FLT3-ITD AML patients than in FLT3 WT patients [45]. CircMYBL2 inhibited apoptosis, enhanced proliferation, and facilitated cell-cycle progression in FLT3-ITD leukemic cells but not FLT3 WT cells. Further, circMYBL2-KD led to reduced FLT3 kinase expression, resulting in modulation of downstream signaling, like reduced phosphorylation of STAT5 (which in turn resulted in reduced expression of $c-M Y C)$. In addition, circMYBL2 enhanced translation of FLT3 kinase by facilitating the polypyrimidine tract-binding protein 1 (PTBP1) binding to FLT3 messenger RNA. In previous studies, FLT3-ITD mutation was associated with increased MCL1 and decreased p27/Kip1 expression, which led to enhanced apoptosis resistance [46, 47]. CircMYBL2-KD was also associated with the reversal of these effects on MCL1 and p27/Kip1 expression levels. In vivo studies demonstrated circMYBL2-KD inhibited FLT3-ITD AML progression and was associated with improved survival in mice with quizartinib sensitive and resistant AML. Overall, these results suggest the effects of driver mutations like FLT3 ITD in AML biology may be mediated (or at least modulated) by altered expression of circRNAs (circ_0000370 and circMYBL2).

In another study, 273 and 296 circRNAs were up and downregulated respectively in pediatric AML patients compared to healthy controls [48]. Circ-004136, produced from the RING finger protein 13 (RNF13) gene at chromosome 3q25.1, was significantly upregulated in pediatric AML patients. Circ-004136 sponges miR142 and miR-29a and regulates AML cell proliferation. Another study also demonstrated upregulation of has_circ_0001346 (circRNF13) in AML patients [49]. In this study, circRNF13 down-regulation was associated with reduced proliferation, cell cycle arrest, increased apoptosis, attenuation of migration, and invasion of AML cells by acting as a sponge for miRNA1224-5p. These effects were mediated by c-MYC regulation at 
Table 1 CircRNAs in acute myeloid leukemia. $\uparrow$ Indicate increased expression, $\downarrow$ Indicate decreased expression. BM (Bone marrow). Ref. (References)

\begin{tabular}{|c|c|c|c|c|c|c|}
\hline $\begin{array}{l}\text { CircRNA } \\
\text { Circ based ID } \\
\text { (Common Name) }\end{array}$ & Host gene & $\begin{array}{l}\text { Sample } \\
\text { (Expression } \\
\text { pattern) }\end{array}$ & Method & Target miRNA/Target gene & Function & Ref. \\
\hline Circ-VIM & Vimentin & $\mathrm{BM}(\uparrow)$ & qRT-PCR & & $\begin{array}{l}\text { Diagnostic/prognostic } \\
\text { biomarker }\end{array}$ & [32] \\
\hline Circ-FOXO3 & $\mathrm{FOXO3}$ & $\mathrm{BM}(\downarrow)$ & qRT-PCR & & Diagnostic biomarker & [33] \\
\hline Hsa_circ_0004277 & WDR37 & $\mathrm{BM}(\downarrow)$ & Microarray/qRT-PCR & & $\begin{array}{l}\text { Diagnostic marker, dynamic } \\
\text { marker with disease status }\end{array}$ & {$[34]$} \\
\hline Circ_0009910 & MFN2 & $\mathrm{BM}(\uparrow)$ & Microarray/qRT-PCR & miR-20a-5p & Prognostic marker & {$[35]$} \\
\hline $\begin{array}{l}\text { Hsa_circ_101141 (Circ- } \\
\text { ANAPC7) }\end{array}$ & ANAPC7 & $\mathrm{BM}(\uparrow)$ & Microarray/qRT-PCR & & & {$[36]$} \\
\hline $\begin{array}{l}\text { Hsa_circ_104700/Hsa_ } \\
\text { circ_0005273 } \\
\text { (Circ-PTK2) }\end{array}$ & & $\mathrm{BM}(\uparrow)$ & $\mathrm{qRT} / \mathrm{PCR}$ & miR-330-5p/FOXM1 & Prognostic marker & {$[37]$} \\
\hline $\begin{array}{l}\text { Hsa_circ_0100181 (Circ- } \\
\text { PAN3) }\end{array}$ & PAN3 & $\mathrm{BM}(\uparrow)$ & Microarray/qRT-PCR & $\begin{array}{l}\text { miR-153-5p, miR-183-5p/ } \\
\text { XIAP }\end{array}$ & Chemoresistance & {$[57,58]$} \\
\hline $\begin{array}{l}\text { Hsa_circ_0035559 (Circ- } \\
\text { ANXA2) }\end{array}$ & ANXA2 & $\mathrm{BM}(\uparrow)$ & Microarray/qRT-PCR & miR-23a-5p and miR-503-3p & $\begin{array}{l}\text { Prognostic biomarker/ } \\
\text { Chemoresistance }\end{array}$ & {$[59]$} \\
\hline $\begin{array}{l}\text { Hsa_circ_0000488 (Cir- } \\
\text { DLEU2) }\end{array}$ & DLEU2 & $\mathrm{BM}(\uparrow)$ & Microarray & miR-496/PRKACB & $\begin{array}{l}\text { Cell proliferation and inhibi- } \\
\text { tion of apoptosis }\end{array}$ & [41] \\
\hline Circ_0000370 & FLI-1 & Blood $(\uparrow)$ & Microarray & miR-1299/S100A7A & Oncogene & {$[44]$} \\
\hline $\begin{array}{l}\text { Hsa_circ_0006332 (Circ- } \\
\text { MYBL2) }\end{array}$ & MYBL2 & $(\uparrow)$ & & $\begin{array}{l}\text { FLT3 translation regulation } \\
\text { through PTPB1 }\end{array}$ & $\begin{array}{l}\text { Oncogene } \\
\text { Therapeutic potential }\end{array}$ & {$[45]$} \\
\hline Hsa_circ_0075001 & NPM1 & $\mathrm{BM}(\uparrow)$ & Microarray & & $\begin{array}{l}\text { Downregulation of Toll-like } \\
\text { receptor signaling }\end{array}$ & {$[54]$} \\
\hline Hsa_circ_0004136 & & $\mathrm{BM}(\uparrow)$ & Microarray/qRT-PCR & miR-142 and miR-29a & Cell proliferation & {$[48]$} \\
\hline $\begin{array}{l}\text { Hsa_circ_0001346 (cir- } \\
\text { cRNF13) }\end{array}$ & RNF13 & Blood $(\uparrow)$ & qRTPCR & miR-1224-5p & $\begin{array}{l}\text { Cell proliferation and migra- } \\
\text { tion }\end{array}$ & [49] \\
\hline Hsa_circ_0121582 & GSK3beta & $\mathrm{BM}(\downarrow)$ & $\begin{array}{l}\text { High-throughput } \\
\text { sequencing/qRT- } \\
\text { PCR }\end{array}$ & miR-224/GSK3beta & $\begin{array}{l}\text { Inhibition of proliferation in } \\
\text { AML cells }\end{array}$ & {$[50]$} \\
\hline Hsa_circ_100,290 & SLC30A7 & $\mathrm{BM}(\uparrow)$ & qRTPCR & Mir-203/Rab10 & $\begin{array}{l}\text { Regulation of proliferation } \\
\text { and apoptosis }\end{array}$ & {$[51]$} \\
\hline Hsa_circ_0079480 & ISPD & $\mathrm{BM}(\uparrow)$ & qRT-PCR & miR-655-3p/HDGF & $\begin{array}{l}\text { Regulation of proliferation } \\
\text { and apoptosis }\end{array}$ & {$[53]$} \\
\hline Hsa_circ_0002483 & & $\mathrm{BM}(\uparrow)$ & qRT-PCR & miR-758-3p/MYC & $\begin{array}{l}\text { Regulation of proliferation, } \\
\text { cell-cycle progression, and } \\
\text { apoptosis }\end{array}$ & {$[52]$} \\
\hline
\end{tabular}

the mRNA level, Caspase $3 / 7$ activation, and reduced Tenascin-C expression at the molecular level. Together these studies suggest circRNA exerts its leukemogenic potential via sponging different miRNAs.

Both the circRNA (hsa_circ_0121582) and its parent gene GSK3beta (a tumor suppressor) are shown to be down-regulated in leukemia cells [50]. Hsa_ circ_0121582 is present both in the cytoplasm and in the nucleus, with different functions evident in each of these compartments: in the cytoplasm, it acts as a sponge for miR-224, and in the nucleus, it binds to the GSK3beta promoter to recruit DNA demethylase TET1. The resultant upregulation of GSK3beta expression inhibits the Wnt/beta-catenin signaling pathway, leading to leukemia cell proliferation.

Other investigators have also demonstrated an association between circRNA and evasion of apoptosis. Hsa circ_100,290 (encoded by parent gene SLC30A7) was upregulated in AML patients [51]. Hsa_circ100290-KD in cell lines inhibited cell proliferation and accelerated apoptosis by targeting miR-203, resulting in regulation of cyclin D1, CDK4, BCL-2, and cleaved Caspase-3 expression, even though expression of host gene SLC30A7 was not affected in this knockout model. It also regulated RAB10 expression, a member of the RAS superfamily of small GTPases, similarly via targeting miR-203. Another 


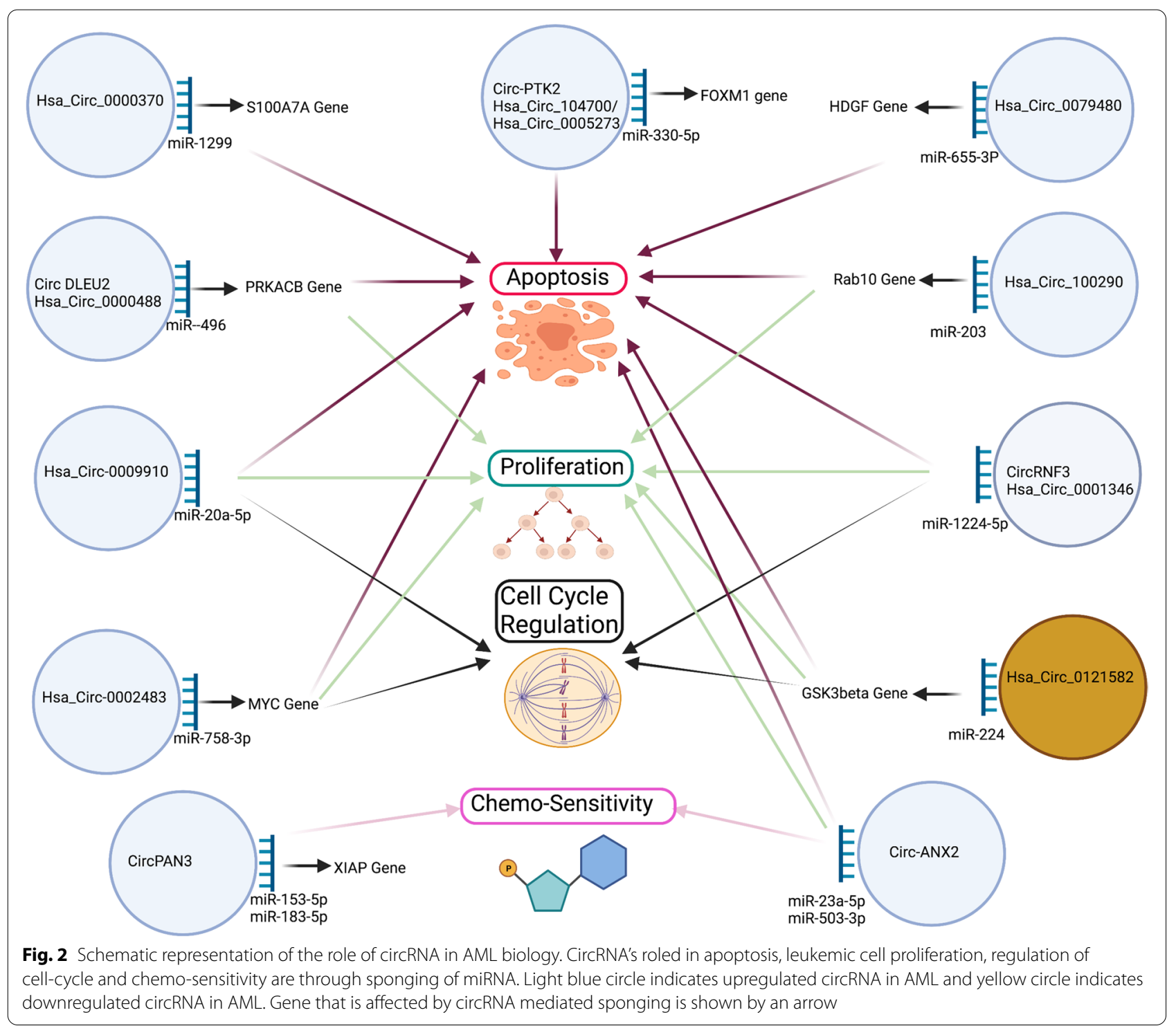

circRNA, hsa_circ_0002483, was overexpressed in AML patients compared to iron-deficient controls [52]. Hsa circ_0002483-KD led to decreased cell proliferation, cell cycle arrest (G0/G1 phase), and increased apoptosis via reduction of $\mathrm{BCL}-2$ and elevation of $\mathrm{BAX}$ and C-caspase-3. It also upregulated MYC expression via sponging miR-758-3p. In another study, hsa_circ_0079480 expression was higher in AML than in idiopathic thrombocytopenia patients [53]. Hsa_circ_0079480-KD was associated with lower viability and increased apoptosis of AML cells. Hsa_circ_0079480 upregulated HDGF (heparin-binding nuclear growth factor) expression via sponging miR-655-3p. In the same study, low miR-655-3p expression and high HDGF expression were associated with poor survival in AML patients.
Whether altered expression of circRNA is sine qua non for AML leukemogenesis, or whether it is simply one of several potentially overlapping abnormalities affecting several steps in AML disease biology remains to be fully determined. Much of the work described herein suggests sponging miRNA and resultant alteration of target gene expression is a central function of circRNA as it relates to leukemogenesis, other mechanisms, including direct binding to the gene promoter regions, need to be further characterized, as well.

\section{The pattern of circRNA expression and AML phenotype}

The historical classification of acute leukemia (FAB) relied on lineage and maturational features of blasts, as reflected by morphological features. A global evaluation 
of circRNA expression from healthy hematopoietic controls and AML patients identified hematopoietic differentiation-associated and AML subgroup-specific signatures [54]. Hsa_circ_0075001, a circRNA of the NPM1 gene, followed a similar expression pattern as the parent NPM1 gene, independent of NPM1 mutation status. High expression of hsa_circ_0075001 associated with no or minimal blast maturation and down-regulation of Toll-like receptor signaling pathway.

Similarly, in another study, whole-transcriptome profiling of 365 younger adults with CN-AML [55] identified 3 distinct circRNA expression-based clusters. These were associated with different clinical and molecular features, e.g., differences in age, extramedullary manifestations, somatic mutations, white blood cell count, platelet count, and blast count. CircFBXW7 was identified to be a regulator of the proliferative capacity of AML blasts. High vs. low expression of circFBXW7 associated with a distinct gene expression signature. No miRNA-binding site was identified for circFBXW7 suggesting that there might be a different mechanism of action than the miRNA sponging described previously. Higher circCFLAR, circKLHL8, circSMC1A, and circFCHO2 expression were each associated with a better prognosis. In each case, except for circFCHO2, correlation with the corresponding linear RNA isoform was weak, indicating observed effects were from circRNA rather than parent gene more generally.

\section{CircRNA and drug resistance in AML}

Primary or secondary refractoriness to treatment (i.e., inherent and acquired drug resistance) is a key limiting factor for optimal outcomes in AML patients. Genetic alterations leading to aberrant activation of drug resistance-related signal pathways is a well-known resistance mechanism in AML [56]. Elucidation of the role of ncRNAs, especially circRNAs, in AML drug resistance, is emerging, potentially providing an avenue for future research developing novel treatment strategies to overcome drug resistance.

Shang et al. studied circRNA expression patterns in doxorubicin (ADM)-resistant THP1 cells and ADM sensitive cells and demonstrated that 49 circRNAs were differentially expressed (35 up- and 14 down-regulated) in doxorubicin (ADM)-resistant THP1 cells compared to ADM sensitive cells [57]. CircPAN3, which was upregulated in resistant cells, was selected for further experiments because of the known role of the parent gene $(P A N 3)$ in leukemogenesis. CircPAN3 was upregulated in relapsed/refractory compared to chemosensitive AML patients, though the parent gene expression did not follow a similar pattern. In cell lines, circPAN3 downregulation was associated with restoring doxorubicin sensitivity, and in contrast, up-regulation was associated with doxorubicin refractoriness. The circPAN3-miR153-5p/miR-183-5p-XIAP (X-linked inhibitor of apoptosis protein) axis was proposed as a mediator of drug resistance. Also, other investigators established circPAN3-mediated autophagy regulation via the AMPK/ mTOR pathway as a resistance mechanism in AML cell lines [58]. Similarly, circ-ANXA2 expression was shown to be upregulated in AML patients [59]. Circ-ANXA2 expression correlated with poor-risk phenotype in AML patients, although multivariable analysis was not performed to discern the independent effects of circ-ANXA2 expression on treatment outcomes. In vitro, circ-ANXA2 knockdown (KD) was associated with increased apoptosis, reduced proliferation of AML cells, and increased chemosensitivity to cytarabine and daunorubicin. It was proposed that circ-ANXA2 possibly worked as a microRNA sponge to dampen the activity of miR-23a-5p and miR-503-3p (as levels of both were increased in circANXA2-KD models). Both miRs are known to increase chemosensitivity [60, 61]. ANXA2 (Annexin A2) expression was not evaluated in this study, but it was proposed that Circ-ANXA2 might enhance transcription of annexin A2, which has a well-established role in leukemogenesis [62, 63].

\section{Profiling circRNA in AML: experimental methods and computational tools}

Structural uniqueness of circRNA can be challenging to study these molecules and it is one of the reasons for being less investigated than other components of the transcriptome. Sequencing of ribosomal RNA (rRNA) depleted total RNA was traditionally used in earlier studies [64]. Though comparative analysis of both linear and circRNAs simultaneously has obvious potential to provide insights to disease pathogenesis, there are technical challenges involved with this. For instance, it can be challenging to choose the level of sequencing depth and dependence on bioinformatics algorithms to identify circRNAs. More commonly used microarray analysis has less reliance on bioinformatics but most published studies have used "RNase R" based treatment for depletion of linear RNAs and hence comparative expression studies are not feasible. To study a specific circRNA of interest, many back-splicing junction (BSJ) locus-specific methods are available, e.g., northern blotting (less popular because of the need for large quantities of RNA and being labor intensive) and reverse transcriptase and quantitative polymerase chain reaction (RT-qPCR, frequently used in circRNA studies). More sensitive but expensive methods including droplet dense polymerase chain recation (ddPCR) and the nCounter platform from NanoString Technologies may provide opportunities for further research $[65,66]$. Both these methods can 
digitally quantify circRNA. In situ hybridization using an oligonucleotide probe that spans the BSJ can identify the circRNA locus within the cell. Various databases have been developed with the help of data obtained from these experimental methods [67]. These databases are commonly used for circRNA identification, quantification, annotation, and network identification to reveal target and downstream signaling. Since the majority of these tools utilize advanced computer programming software, specific training is an absolute requirement.

\section{Limitations of current literature}

The precise mechanism of regulation of circRNA expression in AML is not well studied. Except for fusion circRNAs, it is unclear if dysregulated circRNA expression is a primary event in leukemogenesis or an epiphenomenon. Also, most studies have used bone marrow samples, and only a few had peripheral blood samples. Correlative studies between bone marrow samples and peripheral blood are also lacking.

There is very limited knowledge about the metabolism and transport of circRNA within and outside the cell. It is known that surplus circRNAs are transported out of the cell in exosomes [68]. Although of great interest because of its well elucidated role in multiple other cancers [69], exosomal circRNA has not been studied in AML. Application of exosomal circRNA in modulating bone marrow microenvironment and extramedullary infiltration of leukemia cells can be an attractive area to study.

Relative expression of circRNA and the corresponding parent gene varies and thus may not be regulated in a uniform fashion across the entire genome. In a recent study, the expression of most circRNAs correlated with the expression of the parent gene in both AML patients and healthy controls, but there are instances of discordant expression [54], and also occasional relevant differences in the expression patterns between AML patients and healthy controls. As an example of the latter, circFLT3 expression correlates with parent gene expression only in healthy samples but not in AML patients. Many studies in our review did not examine the circRNA and its parent gene expression simultaneously. Whether any observed effect is from the circRNA or parent gene expression is also not evaluated in most studies, e.g., circVIM is associated with shorter overall survival in AML, but the impact of parent VIM gene expression is not studied. However, both followed a similar expression pattern [32].

Although KD circRNA studies represent a potential opportunity to assess the impact of circRNA expression on that of the parent gene, most studies reviewed herein did not evaluate the circRNA-KD effect on the parent gene expression [38, 41, 44, 48, 49, 52, 53, 59]. Further, much work remains to be done in the area of modeling the interplay of intracellular effects resulting from dysregulation of multiple circRNAs occurring simultaneously.

\section{Conclusion}

AML is a challenging, biologically heterogenous disease with an unmet need for novel diagnostic, therapeutic, and prognostic markers. In this review, we summarized the biological and clinical significance of circRNA in AML. The role ncRNAs, particularly circRNAs, in each of these clinical facets, as well as of leukemogenesis in general is an evolving horizon.

\begin{abstract}
Abbreviations
AML: Acute myeloid leukemia; CircRNA: Circular RNA; ncRNA: non-coding RNA; mRNA: Messenger RNA; RBP: Ribosomal binding protein; miRNA: MicroRNA; RT-qPCR: Reverse transcriptase and quantitative polymerase chain reaction; WBC: White blood cells; FAB: French-American-British; ALL: Acute lymphoblastic lymphoma; MRD: Minimal residual disease; HDGF: heparin-binding nuclear growth factor; RNF13: RING finger protein 13; PTBP1: Polypyrimidine tract-binding protein 1; AUC of ROC: Area under the receiver-operating characteristic curve; CN-AML: Cytogenetically normal AML; KD: Knockdown; EMI: Extramedullary infiltrating; BM: Bone marrow; ADM: Doxorubicin; rRNA: Ribosomal RNA; BSJ: Back splicing junction; ddPCR: Droplet dense polymerase chain reaction.
\end{abstract}

\section{Acknowledgments}

Cancer Center Support Grant P30 (P30CA022453) from NIH is acknowledged. Work in the lab of ASA is supported by R37CA215427 and NIH R01 1R01CA240607. Figure 2 was created with Bio render software.

\section{Authors' contributions}

Vijendra Singh: Conception and design of the study, acquisition, analysis and interpretation of relevant studies, drafting the article and revising it critically for important intellectual content, final approval of the version. Mohammed Hafiz Uddin: Acquisition of data, drafting the article, final approval of the version. Jeffrey Zonder: Conception and design of the study, Drafting the article or revising it critically for important intellectual content, final approval of the version. Asfar S. Azmi: Conception and design of the study, Drafting the article or revising it critically for important intellectual content, final approval of the version. Suresh Balasubramanian: Conception and design of the study, Drafting the article or revising it critically for important intellectual content, final approval of the version.

\section{Funding}

Cancer Center Support Grant P30 (P30CA022453) from NIH is acknowledged. Work in the lab of ASA is supported by R37CA215427 and NIH R01 1R01CA240607.

\section{Availability of data and materials}

Not applicable.

\section{Declarations}

Ethics approval and consent to participate

Not applicable.

Consent for publication

Not applicable.

\section{Competing interests}

The authors declare no direct conflict of interests. Unrelated conflicts are as follows: ASA received funding and speaker fees from Karyopharm Therapeutics Inc. ASA received funding from Janssen, Rhizen, and EISAI. ASA is a consultant for GLG and Guidepoint. 


\section{Author details}

${ }^{1}$ Department of Oncology, Karmanos Cancer Institute/Wayne State University, 4100 John R, HWCRC 740.2, Detroit, MI 48201, USA. ²Department of Oncology, Wayne State University School of Medicine, 4100 John R, HWCRC 732, Detroit, MI 48201, USA.

Received: 2 August 2021 Accepted: 22 October 2021 Published online: 18 November 2021

\section{References}

1. Bennett JM, Catovsky D, Daniel MT, Flandrin G, Galton DA, Gralnick HR, et al. Proposed revised criteria for the classification of acute myeloid leukemia. A report of the French-American-British cooperative group. Ann Intern Med. 1985;103(4):620-5.

2. Arber DA, Orazi A, Hasserjian R, Thiele J, Borowitz MJ, Le Beau MM, et al. The 2016 revision to the World Health Organization classification of myeloid neoplasms and acute leukemia. Blood. 2016;127(20):2391-405.

3. Dohner H, Estey E, Grimwade D, Amadori S, Appelbaum FR, Buchner T, et al. Diagnosis and management of AML in adults: 2017 ELN recommendations from an international expert panel. Blood. 2017;129(4):424-47.

4. Busch W, Kuhnel D, Schirmer K, Scholz S. Tungsten carbide cobalt nanoparticles exert hypoxia-like effects on the gene expression level in human keratinocytes. BMC Genomics. 2010;11:65.

5. Collins $L$, Penny $D$. The RNA infrastructure: dark matter of the eukaryotic cell? Trends Genet. 2009;25(3):120-8.

6. Kung JT, Colognori D, Lee JT. Long noncoding RNAs: past, present, and future. Genetics. 2013;193(3):651-69.

7. Palazzo AF, Lee ES. Non-coding RNA: what is functional and what is junk? Front Genet. 2015;6:2

8. Gao J, Wang F, Wu P, Chen Y, Jia Y. Aberrant LncRNA Expression in Leukemia. J Cancer. 2020;11(14):4284-96.

9. Li Z, Huang C, Bao C, Chen L, Lin M, Wang X, et al. Exon-intron circular RNAs regulate transcription in the nucleus. Nat Struct Mol Biol. 2015;22(3):256-64.

10. Salzman J, Gawad C, Wang PL, Lacayo N, Brown PO. Circular RNAs are the predominant transcript isoform from hundreds of human genes in diverse cell types. PLoS One. 2012;7(2):e30733.

11. Memczak S, Jens M, Elefsinioti A, Torti F, Krueger J, Rybak A, et al. Circular RNAs are a large class of animal RNAs with regulatory potency. Nature. 2013;495(7441):333-8.

12. Zhang $Y$, Zhang $X O$, Chen $T$, Xiang JF, Yin QF, Xing YH, et al. Circular intronic long noncoding RNAs. Mol Cell. 2013;51(6):792-806.

13. Salzman J, Chen RE, Olsen MN, Wang PL, Brown PO. Cell-type specific features of circular RNA expression. PLoS Genet. 2013;9(9):e1003777.

14. Guarnerio J, Bezzi M, Jeong JC, Paffenholz SV, Berry K, Naldini MM, et al. Oncogenic role of fusion-circRNAs derived from Cancer-associated chromosomal translocations. Cell. 2016;165(2):289-302.

15. Jeck WR, Sorrentino JA, Wang K, Slevin MK, Burd CE, Liu J, et al. Circular RNAs are abundant, conserved, and associated with ALU repeats. RNA. 2013;19(2):141-57.

16. Rybak-Wolf A, Stottmeister C, Glazar P, Jens M, Pino N, Giusti S, et al. Circular RNAs in the mammalian brain are highly abundant, conserved, and dynamically expressed. Mol Cell. 2015;58(5):870-85.

17. Xu S, Zhou L, Ponnusamy M, Zhang L, Dong Y, Zhang Y, et al. A comprehensive review of circRNA: from purification and identification to disease marker potential. PeerJ. 2018;6:e5503.

18. Thomson DW, Dinger ME. Endogenous microRNA sponges: evidence and controversy. Nat Rev Genet. 2016;17(5):272-83.

19. Chen N, Zhao G, Yan X, Lv Z, Yin H, Zhang S, et al. A novel FLI1 exonic circular RNA promotes metastasis in breast cancer by coordinately regulating TET1 and DNMT1. Genome Biol. 2018;19(1):218.

20. Dudekula DB, Panda AC, Grammatikakis I, De S, Abdelmohsen K, Gorospe M. Circlnteractome: a web tool for exploring circular RNAs and their interacting proteins and microRNAs. RNA Biol. 2016;13(1):34-42.

21. Zeng Y, Du WW, Wu Y, Yang Z, Awan FM, Li X, et al. A circular RNA binds to and activates AKT phosphorylation and nuclear localization reducing apoptosis and enhancing cardiac repair. Theranostics. 2017:7(16):3842-55.
22. Pamudurti NR, Bartok O, Jens M, Ashwal-Fluss R, Stottmeister C, Ruhe L, et al. Translation of CircRNAs. Mol Cell. 2017;66(1):9-21 e7.

23. Yang $Y$, Fan $X$, Mao M, Song X, Wu P, Zhang Y, et al. Extensive translation of circular RNAs driven by N(6)-methyladenosine. Cell Res. 2017;27(5):626-41.

24. Wilusz JE, Sunwoo H, Spector DL. Long noncoding RNAs: functional surprises from the RNA world. Genes Dev. 2009;23(13):1494-504.

25. Bhat SA, Ahmad SM, Mumtaz PT, Malik AA, Dar MA, Urwat U, et al. Long non-coding RNAs: mechanism of action and functional utility. Noncoding RNA Res. 2016;1(1):43-50.

26. Han B, Chao J, Yao H. Circular RNA and its mechanisms in disease: from the bench to the clinic. Pharmacol Ther. 2018;187:31-44.

27. Zhang Z, Yang T, Xiao J. Circular RNAs: promising biomarkers for human diseases. EBioMedicine. 2018;34:267-74.

28. Bach DH, Lee SK, Sood AK. Circular RNAs in Cancer. Mol Ther Nucleic Acids. 2019:16:118-29.

29. Bahn JH, Zhang Q, Li F, Chan TM, Lin X, Kim Y, et al. The landscape of microRNA, Piwi-interacting RNA, and circular RNA in human saliva. Clin Chem. 2015;61(1):221-30.

30. Li Y, Zheng Q, Bao C, Li S, Guo W, Zhao J, et al. Circular RNA is enriched and stable in exosomes: a promising biomarker for cancer diagnosis. Cell Res. 2015;25(8):981-4

31. Memczak S, Papavasileiou P, Peters O, Rajewsky N. Identification and characterization of circular RNAs as a new class of putative biomarkers in human blood. PLoS One. 2015;10(10):e0141214.

32. Yi YY, Yi J, Zhu X, Zhang J, Zhou J, Tang X, et al. Circular RNA of vimentin expression as a valuable predictor for acute myeloid leukemia development and prognosis. J Cell Physiol. 2019;234(4):3711-9.

33. Zhou J, Zhou LY, Tang X, Zhang J, Zhai LL, Yi YY, et al. Circ-Foxo3 is positively associated with the Foxo3 gene and leads to better prognosis of acute myeloid leukemia patients. BMC Cancer. 2019;19(1):930.

34. Li W, Zhong C, Jiao J, et al. Characterization of hsa_circ_0004277 as a New Biomarker for Acute Myeloid Leukemia via Circular RNA Profile and Bioinformatics Analysis. Int J Mol Sci. 2017;18(3):597.

35. Ping L, Jian-Jun C, Chu-Shu L, Guang-Hua L, Ming Z. Silencing of circ_0009910 inhibits acute myeloid leukemia cell growth through increasing miR-20a-5p. Blood Cells Mol Dis. 2019;75:41-7.

36. Chen H, Liu T, Liu J, Feng Y, Wang B, Wang J, et al. Circ-ANAPC7 is Upregulated in acute myeloid leukemia and appears to target the MiR-181 family. Cell Physiol Biochem. 2018;47(5):1998-2007.

37. Lv C, Sun L, Guo Z, Li H, Kong D, Xu B, et al. Circular RNA regulatory network reveals cell-cell crosstalk in acute myeloid leukemia extramedullary infiltration. J Transl Med. 2018;16(1):361.

38. Yi L, Zhou L, Luo J, Yang Q. Circ-PTK2 promotes the proliferation and suppressed the apoptosis of acute myeloid leukemia cells through targeting miR-330-5p/FOXM1 axis. Blood Cells Mol Dis. 2021;86:102506.

39. Guo S, Li B, Chen Y, Zou D, Yang S, Zhang Y, et al. Hsa_circ_0012152 and Hsa_circ_0001857 accurately discriminate acute lymphoblastic leukemia from acute myeloid leukemia. Front Oncol. 2020;10:1655.

40. Testa U, Riccioni R. Deregulation of apoptosis in acute myeloid leukemia. Haematologica. 2007;92(1):81-94.

41. Wu DM, Wen X, Han XR, et al. Role of Circular RNA DLEU2 in Human Acute Myeloid Leukemia. Mol Cell Biol. 2018;38(20):e00259-18.

42. Morenos L, Chatterton Z, Ng JL, Halemba MS, Parkinson-Bates M, Mechinaud F, et al. Hypermethylation and down-regulation of DLEU2 in paediatric acute myeloid leukaemia independent of embedded tumour suppressor miR-15a/16-1. Mol Cancer. 2014;13:123.

43. Kasar S, Underbayev C, Yuan Y, Hanlon M, Aly S, Khan H, et al. Therapeutic implications of activation of the host gene (Dleu2) promoter for miR-15a/16-1 in chronic lymphocytic leukemia. Oncogene. 2014;33(25):3307-15

44. Zhang L, Bu Z, Shen J, Shang L, Chen Y, Wang Y. A novel circular RNA (hsa_circ_0000370) increases cell viability and inhibits apoptosis of FLT3ITD-positive acute myeloid leukemia cells by regulating miR-1299 and S100A7A. Biomed Pharmacother. 2020;122:109619.

45. Sun YM, Wang WT, Zeng ZC, Chen TQ, Han C, Pan Q, et al. circMYBL2, a circRNA from MYBL2, regulates FLT3 translation by recruiting PTBP1 to promote FLT3-ITD AML progression. Blood. 2019;134(18):1533-46.

46. Yoshimoto G, Miyamoto T, Jabbarzadeh-Tabrizi S, lino T, Rocnik JL, Kikushige $Y$, et al. FLT3-ITD up-regulates MCL-1 to promote survival of 
stem cells in acute myeloid leukemia via FLT3-ITD-specific STAT5 activation. Blood. 2009;114(24):5034-43.

47. Scheijen B, Ngo HT, Kang H, Griffin JD. FLT3 receptors with internal tandem duplications promote cell viability and proliferation by signaling through Foxo proteins. Oncogene. 2004;23(19):3338-49.

48. Yuan DM, Ma J, Fang WB. Identification of non-coding RNA regulatory networks in pediatric acute myeloid leukemia reveals circ-0004136 could promote cell proliferation by sponging miR-142. Eur Rev Med Pharmacol Sci. 2019;23(21):9251-8.

49. Zhang R, Li Y, Wang H, Zhu K, Zhang G. The regulation of circRNA RNF13/ miRNA-1224-5p Axis promotes the malignant evolution in acute myeloid leukemia. Biomed Res Int. 2020;2020:5654380.

50. Chen JJ, Lei P, Zhou M. hsa_circ_0121582 inhibits leukemia growth by dampening Wnt/beta-catenin signaling. Clin Transl Oncol. 2020;22(12):2293-302.

51. Fan H, Li Y, Liu C, Liu Y, Bai J, Li W. Circular RNA-100290 promotes cell proliferation and inhibits apoptosis in acute myeloid leukemia cells via sponging miR-203. Biochem Biophys Res Commun. 2018;507(1-4):178-84

52. Xiao Y, Ming X, Wu J. Hsa_circ_0002483 regulates miR-758-3p/MYC axis to promote acute myeloid leukemia progression. Hematol Oncol. 2021;39(2):243-53.

53. Hu Q, Gu Y, Chen S, Tian Y, Yang S. Hsa_circ_0079480 promotes tumor progression in acute myeloid leukemia via miR-654-3p/HDGF axis. Aging (Albany NY). 2020;13(1):1120-31. https://doi.org/10.18632/aging.202240.

54. Hirsch S, Blatte TJ, Grasedieck S, Cocciardi S, Rouhi A, Jongen-Lavrencic M, et al. Circular RNAs of the nucleophosmin (NPM1) gene in acute myeloid leukemia. Haematologica. 2017;102(12):2039-47.

55. Papaioannou D, Volinia S, Nicolet D, Swierniak M, Petri A, Mrozek K, et al. Clinical and functional significance of circular RNAs in cytogenetically normal AML. Blood Adv. 2020:4(2):239-51.

56. Kumar CC. Genetic abnormalities and challenges in the treatment of acute myeloid leukemia. Genes Cancer. 2011;2(2):95-107.

57. Shang J, Chen WM, Wang ZH, Wei TN, Chen ZZ, Wu WB. CircPAN3 mediates drug resistance in acute myeloid leukemia through the miR-153-5p/ miR-183-5p-XIAP axis. Exp Hematol. 2019;70:42-54 e3.

58. Shang J, Chen WM, Liu S, Wang ZH, Wei TN, Chen ZZ, et al. CircPAN3 contributes to drug resistance in acute myeloid leukemia through regulation of autophagy. Leuk Res. 2019;85:106198.

59. Ding Y, Dong Y, Lu H, Luo X, Fu J, Xiu B, et al. Circular RNA profile of acute myeloid leukaemia indicates circular RNA annexin A2 as a potential biomarker and therapeutic target for acute myeloid leukaemia. Am J Transl Res. 2020;12(5):1683-99.

60. Ganesan S, Palani HK, Lakshmanan V, Balasundaram N, Alex AA, David $\mathrm{S}$, et al. Stromal cells downregulate miR-23a-5p to activate protective autophagy in acute myeloid leukemia. Cell Death Dis. 2019;10(10):736.

61. Seo M, Kim SM, Woo EY, Han KC, Park EJ, Ko S, et al. Stemness-attenuating miR-503-3p as a paracrine factor to regulate growth of Cancer stem cells. Stem Cells Int. 2018;2018:4851949.

62. Bharadwaj A, Bydoun M, Holloway R, Waisman D. Annexin A2 heterotetramer: structure and function. Int J Mol Sci. 2013;14(3):6259-305.

63. Huang D, Yang Y, Sun J, Dong X, Wang J, Liu H, et al. Annexin A2-S100A10 heterotetramer is upregulated by PML/RARalpha fusion protein and promotes plasminogen-dependent fibrinolysis and matrix invasion in acute promyelocytic leukemia. Front Med. 2017;11(3):410-22.

64. Glazar P, Papavasileiou P, Rajewsky N. circBase: a database for circular RNAs. RNA. 2014;20(11):1666-70.

65. Geiss GK, Bumgarner RE, Birditt B, Dahl T, Dowidar N, Dunaway DL, et al. Direct multiplexed measurement of gene expression with color-coded probe pairs. Nat Biotechnol. 2008;26(3):317-25.

66. LiT, Shao Y, Fu L, Xie Y, Zhu L, Sun W, et al. Plasma circular RNA profiling of patients with gastric cancer and their droplet digital RT-PCR detection. J Mol Med (Berl). 2018;96(1):85-96.

67. Chen L, Wang C, Sun H, Wang J, Liang Y, Wang Y, et al. The bioinformatics toolbox for circRNA discovery and analysis. Brief Bioinform. 2021;22(2):1706-28.

68. Lasda E, Parker R. Circular RNAs co-precipitate with extracellular vesicles: a possible mechanism for circRNA clearance. PLoS One. 2016;11(2):e0148407.

69. Seimiya T, Otsuka M, Iwata T, Shibata C, Tanaka E, Suzuki T, et al. Emerging roles of Exosomal circular RNAs in Cancer. Front Cell Dev Biol. 2020:8:568366.

70. Kristensen LS, Andersen MS, Stagsted LVW, Ebbesen KK, Hansen TB, Kjems J. The biogenesis, biology and characterization of circular RNAs. Nat Rev Genet. 2019;20(11):675-91.

\section{Publisher's Note}

Springer Nature remains neutral with regard to jurisdictional claims in published maps and institutional affiliations.
Ready to submit your research? Choose BMC and benefit from:

- fast, convenient online submission

- thorough peer review by experienced researchers in your field

- rapid publication on acceptance

- support for research data, including large and complex data types

- gold Open Access which fosters wider collaboration and increased citations

- maximum visibility for your research: over $100 \mathrm{M}$ website views per year

At BMC, research is always in progress.

Learn more biomedcentral.com/submissions 